10

\title{
Влияние конфигурации и материала встречно-штыревых преобразователей на возбуждение поверхностных и псевдоповерхностных акустических волн в подложках ниобата лития
}

\author{
(C) А.В. Варламов ${ }^{1,2}$, В.В. Лебедев ${ }^{1}$, П.М. Агрузов ${ }^{1}$, И.В. Ильичёв ${ }^{1}$, А.В. Шамрай ${ }^{1}$ \\ ${ }^{1}$ Физико-технический институт им. А.Ф. Иоффре РАН, \\ Санкт-Петербург, Россия \\ ${ }^{2}$ Санкт-Петербургский политехнический университет Петра Великого, \\ Санкт-Петербург, Россия \\ E-mail: wwa@bk.ru
}

Поступило в Редакцию 22 февраля 2019 г.

В окончательной редакции 22 февраля 2019 г.

Принято к публикации 16 апреля 2019 г.

Исследованы возбуждение, распространение и взаимодействие поверхностных и псевдоповерхностных акустических волн в подложке ниобата лития $X$-среза. Определены резонансные частоты возбуждения, скорости распространения и дисперсионные характеристики для каждого типа акустических волн. Выявлено влияние материала электродов на эффективность возбуждения и взаимодействие между разными типами акустических волн. Определены требования к материалу и конфигурации электродов акустооптических устройств в интегральном исполнении.

Ключевые слова: поверхностная акустическая волна, псевдоповерхностная акустическая волна, встречноштыревой преобразователь, акустооптическое устройство, ниобат лития, интегральная оптика.

DOI: 10.21883/PJTF.2019.14.48023.17749

Поверхностные акустические волны (ПАВ) широко используются для создания акустооптических устройств в интегральном исполнении $[1,2]$. Локализация оптического излучения в оптическом волноводе и ПАВ на поверхности подложки обеспечивают высокую эффективность акустооптического взаимодействия и низкую мощность управляющих высокочастотных электрических (ВЧ) сигналов. Ниобат лития (НЛ) благодаря своим пьезоэлектрическим свойствам и отработанной промышленной технологии изготовления оптических волноводов является основным материалом для создания акустооптических модуляторов в интегральном исполнении [3]. Обычно в качестве критерия оптимизации таких устройств выступает эффективность акустооптического преобразования [4-6]. В настоящей работе мы сконцентрировались на подавлении возбуждения паразитных псевдоповерхностных акустических волн (псевдо- ПАВ, вытекающие ПАВ) [7,8]. Возбуждение паразитных псевдо-ПАВ, их взаимодействие с оптическим излучением и ПАВ приводят не только к снижению эффективности акустооптического преобразования, но и к дополнительным шумам в оптическом тракте, что особенно критично для волоконно-оптических датчиков [9] (основной области применения интегральной акустооптики).

Цель настоящей работы - исследовать влияние конфигурации и материала встречно-штыревых преобразователей (ВШП) на возбуждение, распространение и взаимодействие ПАВ и псевдо-ПАВ в подложках НЛ и сформулировать требования к конструкции электродов интегральных акустооптических устройств.
Была выбрана конфигурация интегрально-оптического модулятора сдвига частоты (рис. 1), представляющего собой монокристаллическую пластину НЛ $X$-среза, на поверхности которой методом термической диффузии титана [10] вдоль кристаллографического направления $Y$ сформирован канальный оптический волновод (одномодовый для $1550 \mathrm{~nm}$ ). ВШП были изготовлены на поверхности волновода методом магнетронного напыления. Расстояние между центрами штырей $10 \mu \mathrm{m}$ соответствовало возбуждению акустических волн в диапазоне частот 150-250 MHz. Было изготовлено несколько образцов с ВШП из разного материала и с разным количеством штырей (см. таблицу). Образцы были состыкованы с оптическим волокном методом приклейки в торец. Области стыковки одновременно служили поглотителями ПАВ и обеспечивали для них режим бегущей волны.

На первом этапе были исследованы частотные зависимости проводимости ВШП. Максимумы на зависимостях действительной части проводимости соответствуют резонансному возбуждению акустических волн и преобразованию электрического сигнала в акустические колебания (рис. 2). Зависимости должны описываться формулой [11]:

$$
\operatorname{Re}(Y(f))=\hat{G}_{a}\left(\frac{\sin \left(\frac{N}{2} \pi\left(\frac{f-f_{0}}{f_{0}}\right)\right)}{\frac{N}{2} \sin \left(\pi\left(\frac{f-f_{0}}{f_{0}}\right)\right)}\right)^{2},
$$

где $f_{0}$ - резонансная частота генерации акустической волны, $N$ - количество штырей ВШП, $\hat{G}_{a}-$ множи- 


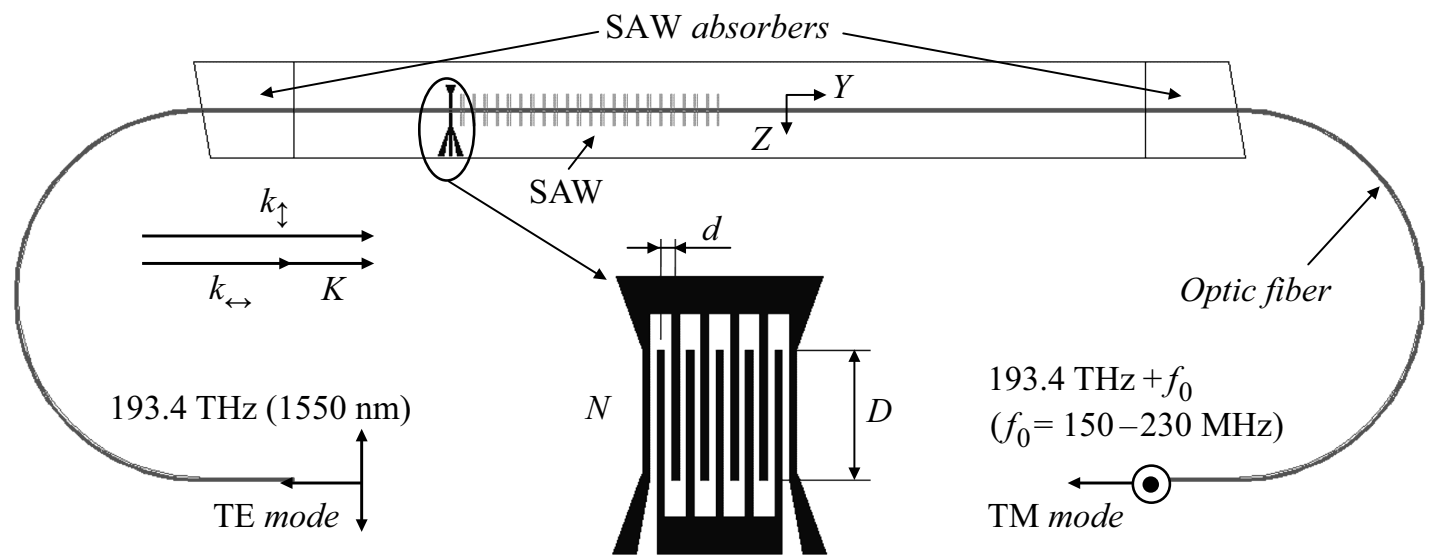

Pис. 1. Акустооптический модулятор сдвига частоты в интегральном исполнении и конфигурация ВШП $(D=1.7 \mathrm{~mm}-$ апертура, $d=10 \mu \mathrm{m}$ - расстояние между центрами соседних электродов, $N=11$ или 17 - количество штырей).
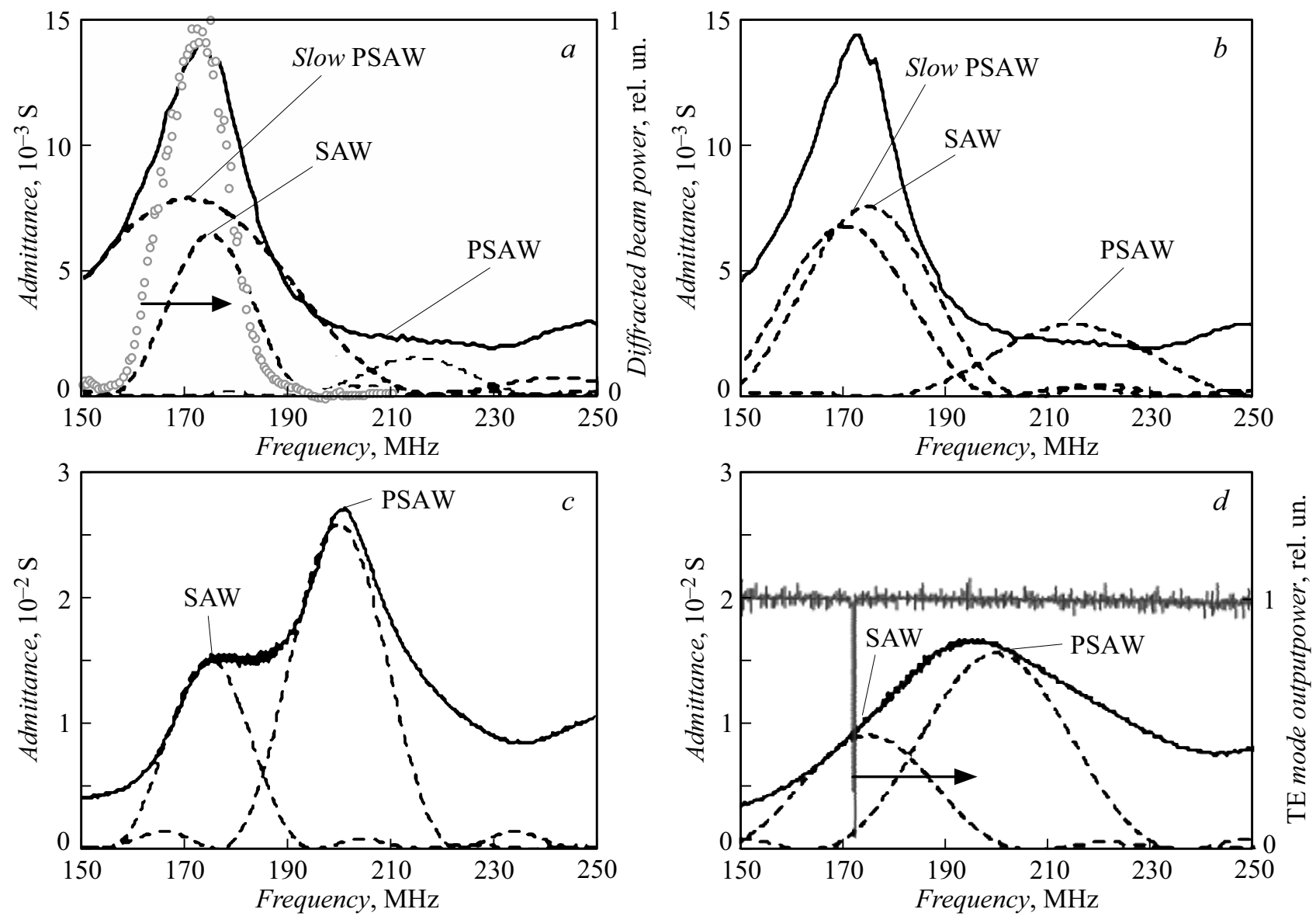

Рис. 2. Экспериментальные (сплошные линии) и теоретические (штриховые линии) частотные характеристики действительных частей проводимостей Au-17 $(a), \mathrm{Au}-11(b), \mathrm{Al}-17(c), \mathrm{Al}-11(d)$. Результаты дифракционных измерений на образце Аu-17 (правая шкала на части $a$ ), частотная зависимость акустооптического преобразования поляризации внутри оптического волновода (правая шкала на части $d$ ).

тель, зависящий от направления электрического поля относительно направления распространения акустической волны. Для конфигурации экспериментальных образцов

$$
\hat{G}_{a}=\frac{\pi^{2} C_{T} f_{0}}{k^{2} N}
$$

где $k^{2}-$ квадрат коэффициента электромеханической связи НЛ, $C_{T}$ - электрическая емкость ВШП. Однако экспериментальные зависимости плохо описываются выражением (1) для возбуждения только бегущей ПАВ. Ширина резонансных пиков значительно больше, а для образца с наибольшим числом алюминиевых штырей 
Параметры возбуждаемых акустических волн

\begin{tabular}{c|c|c|c|c}
\hline Конфигурации ВшП & Параметр & ПАВ & Псевдо-ПАВ & $\begin{array}{c}\text { Замедленная } \\
\text { псевдо-ПАВ }\end{array}$ \\
\hline Аи-17, Аu-11 & Резонансная частота, $\mathrm{MHz}$ & 175 & 215 & 171 \\
(материал & Угол фазового фронта, & 0 & 20 & 20 \\
хром-золото, & Скорость волны, $\mathrm{m} / \mathrm{s}$ & 3720 & 4550 & 3625 \\
17 и 11 штырей) & $k^{2}$ & 0.048 & 0.034 & 0.034 \\
\hline Аl-17, Аl-11 & Резонансная частота, $\mathrm{MHz}$ & 175 & 200 & - \\
(материал & Угол фазового фронта, & 0 & 20 & - \\
алюминий, & Скорость волны, $\mathrm{m} / \mathrm{s}$ & 3560 & 4620 & -
\end{tabular}

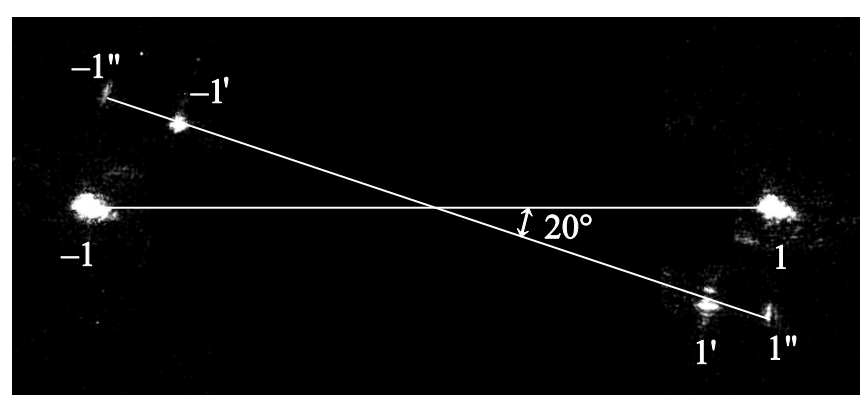

Рис. 3. Картина дифракции на акустических волнах для образца Au-17. 1 и -1 - первый и минус первый порядки дифракции на ПАВ, $1^{\prime}$ и $-1^{\prime}-$ первый и минус первый порядки дифракции на псевдо-ПАВ, $1^{\prime \prime}$ и $-1^{\prime \prime}$ - первый и минус первый порядки дифракции на повернутой ПАВ, возбужденной замедленной псевдо-ПАВ.

наблюдается дополнительный максимум на более высокой частоте, чем частота возбуждения ПАВ. Кроме того, зависимости существенным образом отличались для ВШП из алюминия и золота. Было сделано предположение о дополнительном возбуждении другого вида акустических волн - так называемых псевдо-ПАВ.

Для подтверждения данного предположения были проведены исследования дифракции отраженного пробного лазерного пучка видимого диапазона $(532 \mathrm{~nm})$, нормально падающего на различные участки поверхности образцов, в области ВШП и в области свободного распространения акустических волн. На картине дифракции (рис. 3) помимо дифракционных порядков, соответствующих ПАВ, наблюдались дифракционные порядки от других акустических волн. Ярко выражен порядок дифракции на псевдо-ПАВ с фазовым фронтом, повернутым относительно фазового фронта ПАВ, задаваемого ВШП, приблизительно на 20․ Максимальная интенсивность дифракционных порядков от ПАВ и от псевдоПАВ наблюдалась при разных значениях частоты. Из угла дифракции и значения частоты возбуждения были вычислены скорости распространения ПАВ и псевдоПАВ (см. таблицу). Отметим, что в образцах с золотыми электродами угол дифракции на псевдо-ПАВ в области ВШП был близок к углу дифракции на ПАВ, так как более тяжелые и пластичные золотые электроды приводят к замедлению псевдо-ПАВ в области ВШП. Согласование скоростей ПАВ и замедленной псевдоПАВ приводит к возбуждению дополнительной ПАВ с фазовым фронтом, повернутым относительно ВШП приблизительно на $20^{\circ}$. Повернутая ПАВ и замедленная псевдо-ПАВ эффективно взаимодействуют, что наблюдалось в виде противофазного мерцания дифракционных порядков ПАВ и псевдо-ПАВ при изменении частоты возбуждения.

По результатам дифракционных исследований в модель, описывающую частотную зависимость проводимости ВШП, помимо вклада от ПАВ $\left(Y_{\mathrm{SAW}}\right)$ были включены дополнительные вклады от псевдо-ПАВ ( $\left.Y_{\mathrm{PSAW}}\right)$ и замедленной псевдо-ПАВ ( $Y_{\text {slow }}$ PSAW $)$

$$
\begin{aligned}
\operatorname{Re}(Y(f))= & \operatorname{Re}\left(Y_{\mathrm{SAW}}(f)\right)+\operatorname{Re}\left(Y_{\text {PSAW }}(f)\right) \\
& +\left\{\operatorname{Re}\left(Y_{\text {slow } \operatorname{PSAW}}(f)\right)\right\} .
\end{aligned}
$$

Составляющая проводимости для каждого вида акустических волн описывается выражениями (1) и (2) с резонансными частотами возбуждения, определенными с помощью дифракционных экспериментов (см. таблицу), и коэффициентами электромеханической связи, использующимися в качестве подгоночных параметров. Теоретические частотные зависимости проводимости, учитывающие возбуждение псевдо-ПАВ и замедленной псевдо-ПАВ, хорошо согласуются с экспериментальными данными (рис. 2). Для алюминиевого ВШП с 17 штырями появление двойного максимума обусловлено сужением резонансных пиков, соответствующих различным видам акустических волн, что дает возможность их разрешения на общей частотной зависимости проводимости. Значительный вклад замедленной псевдо-ПАВ наблюдается у золотого ВШП. При этом резонансная частота возбуждения замедленной псевдо-ПАВ близка к частоте возбуждения ПАВ, из-за чего на результирующей характеристике проводимости эти два пика сливаются. Отметим, что в этом случае взаимодействие замедленной псевдо-ПАВ и ПАВ может приводить к дополнительным шумам в оптическом тракте модулятора. На алюминиевых электродах вкладом замедленной псевдо-ПАВ можно пренебречь, и соответственно 
влияние псевдо-ПАВ на оптический сигнал в волноводе пренебрежимо мало и связано только со снижением эффективности возбуждения ПАВ за счет потери энергии при уходе псевдо-ПАВ с поверхности в глубину кристаллической подложки. Это было подтверждено измерениями частотной зависимости акустооптического преобразования поляризации внутри оптического волновода на длине волны $1550 \mathrm{~nm}$ при мощности модулирующего сигнала $100 \mathrm{~mW}$. На частоте возбуждения ПАВ $(172.3 \mathrm{MHz})$ наблюдался узкий пик (рис. 2,d), соответствующий преобразованию волноводной моды с ТЕполяризацией в моду с ТМ-поляризацией. На частотах возбуждения псевдо-ПАВ влияние на оптический сигнал не наблюдается.

Таким образом, выявлено, что помимо модулирующей оптический сигнал ПАВ значительная энергия электрического ВЧ-сигнала может уходить на возбуждение паразитных псевдо-ПАВ и замедленной псевдо-ПАВ. Для ВШП, изготовленного из золота, частота резонансного возбуждения замедленной псевдо-ПАВ близка к частоте возбуждения ПАВ, что приводит к их взаимодействию и служит источником дополнительных шумов в оптическом сигнале. Это является даже более важным аргументом в пользу выбора алюминия для изготовления электродов интегральных акустооптических модуляторов, чем эффективность возбуждения ПАВ. Для алюминиевых ВШП потери на возбуждение псевдоПАВ могут быть минимизированы за счет увеличения количества штырей, однако при этом будет сужаться частотная полоса модулирующих ВЧ-сигналов. Оптимальная конфигурация ВШП должна быть согласована с характеристиками модулирующего сигнала.

\section{Конфликт интересов}

Авторы заявляют, что у них нет конфликта интересов.

\section{Список литературы}

[1] Morgan D. Surface acoustic wave filters: with applications to electronic communications and signal processing. 2nd ed. Amsterdam: Elsevier, 2007. 429 p.

[2] Bo L., Xiao C., Hualin C., Mohammad M.A., Xiangguang T., Luqi T., Yi Y., Tianling R. // J. Semicond. 2016. V. 37. N 2. P. 021001(1-9).

[3] Lejman M., Vaudel G., Infante I.C., Chaban I., Pezeril T., Edely M., Nataf G.F., Guennou M., Kreisel J., Gusev V.E., Dkhil B., Ruello P. // Nature Commun. 2016. V. 7. N 1. P. $12345(1-10)$.

[4] Ward J.D., Valle S., Pannell C., Johnson N.P. // J. Phys.: Conf. Ser. 2015. V. 619. P. 012054(1-4).

[5] Liu T., Yu K. Collinear surface acoustic wave acousto-electrooptic modulator in $\mathrm{Ti}_{\mathrm{LiNbO}}$ waveguide // 2010 Symp. on photonics and optoelectronics. IEEE, 2010. P. 1-3.

[6] Varlamov A.V., Shamray A.V., Lebedev V.V., Agrusov P.M., Ilichev I.V. Search for optimal conditions of SAW excitation by lithium niobate integrated optical TE-TM mode convertor // 2018 IEEE Int. Conf. on electrical engineering and photonics (EExPolytech). IEEE, 2018. P. 172-175.
[7] Kakio S. // Acta Phys. Pol. A. 2015. V. 127. N 1. P. 15-19.

[8] Peverini O.A., Orta R., Tascone R. // Opt. Quant. Electron. 2000. V. 32. N 6-8. P. 855-867.

[9] Yin S., Ruffin P.B., Yu F.T.S. Fiber optic sensors. 2nd ed. Boca Raton: CRC Press, 2008. 477 p.

[10] Караваев П.М., Ильччев И.В., Агрузов П.М., Тронев А.В., Шамрай А.В. // Письма в ЖТФ. 2016. Т. 42. В. 10. С. 3339.

[11] Smith W.R., Gerard H.M., Collins J.H., Reeder T.M., Shaw H.J. // IEEE Transact. Microwave Theory Techn. 1969. V. 17. N 11. P. 856-864. 